\title{
AN ac IMPEDANCE STUDY OF DENDRITIC SILVER THREE-DIMENSIONAL ELECTRODEPOSITS
}

\author{
A. Hernández-Creus, ${ }^{*}$ A. E. Bolzán, $\dagger$ P. Carro, ${ }^{*}$ S. González,* R. C. Salvarezza $\dagger$ \\ and A. J. ARviat \\ * Departamento de Química-Física, Universidad de la Laguna, Tenerife, Spain; \\ † Instituto de Investigaciones Fisicoquímicas Teóricas y Aplicadas, (INIFTA), La Plata, Argentina
}

(Received 13 October 1992; in revised form 25 January 1993)

\begin{abstract}
The ac impedance behaviour of electrochemically grown dendritic Ag three-dimensional electrodeposits grown from aqueous $\mathrm{Ag}^{+}$ion containing solutions has been studied in the blocking regime covering the $1 \leqslant f \leqslant 10^{4} \mathrm{~Hz}$ frequency range $(\omega=2 \pi f)$. These deposits exhibit fractal surface and non fractal mass (volume) characteristics. Impedance data displayed as $\log Z^{\prime \prime}$ vs. $\log \omega$ plots show two linear portions with different frequency dispersion exponents $\langle\alpha\rangle=0.95 \pm 0.03$ and $\langle\alpha\rangle=0.67 \pm 0.05$, respectively. The crossover frequency, $\omega_{\lambda}$, as determined from those plots, mainly depends on the electrolyte resistivity and, to a minor extent, on the $\mathrm{Ag}^{+}$ion electrodeposition charge. For $\omega>\omega_{\lambda}$, the surface fractal dimension derived from the reciprocal relationship, $D_{s}=(1 / \alpha)+1$, is $D_{s}=2.45 \pm 0.1$. This figure agrees with that obtained from the $\log$ (area) vs. $\log$ (volume) plots.
\end{abstract}

Key words: fractals, Ag electrodes, rough metal surfaces, ac impedance, electrodeposition.

\section{INTRODUCTION}

The possibility of evaluating the fractal dimension of rough metal surfaces by the $a c$ impedance technique has attracted a considerable interest over the past years [1-5]. The following relationship between $Z^{\prime \prime}$, the electrode impedance, and $\omega$, the frequency, is fulfilled for these surfaces in the blocking regime:

$$
Z^{\prime \prime} \propto j \omega^{-a}
$$

where $j=\sqrt{-1}, \omega=2 \pi f, f$ is the frequency of the ac signal in $\mathrm{Hz}$ and $\alpha$ is the frequency dispersion exponent, denoted as the constant phase angle (CPA). For a perfectly smooth metal surface showing ideal capacitive behaviour $\alpha=1$; whereas for irregular surfaces in diluted solutions $\alpha<1[1,6]$.

Although different models have been proposed to relate $\alpha$ to $D_{3}$, the fractal dimension of the metal electrode surface, no reliable relationship between these two parameters could be established as yet[711]. However, in a recent approach to this problem, it was found that for a diffusion limited aggregation (DLA) type electrode, the following equation holds [12]:

$$
D_{\mathrm{a}}=(1 / \alpha)+1 .
$$

Equation (2) offers the possibility of evaluating $D_{\mathrm{s}}$ through analysis of the impedance diagrams.

ac impedance data of $\mathbf{A g}$ dendritic threedimensional electrodeposits are reported in the present work. The surface of these deposits can be described in terms of DLA-type metallic surface[13]. These data have fulfilled equation[1] over about three decades of $\omega$ with either $\alpha \cong 1$ or $\alpha \cong 0.67$, depending on whether $\omega$ is lower or greater than a certain crossover frequency. From the $\alpha$ value found for $\omega$ greater than the crossover frequency, it results in $D_{\mathrm{s}}=2.45 \pm 0.10$, a figure which agrees with the value of $D_{\mathrm{a}}$ derived from the analysis of the $\log$ (area) vs. $\log$ (volume) plots[13]. Accordingly, the $D_{s}$ value for $\mathbf{A g}$ dendrites electrodeposits agrees with the prediction for three-dimensional deposits generated from DLA models[14].

\section{EXPERIMENTAL}

The $\mathrm{Ag}$ dendritic electrodeposits were grown under potentiostatic conditions on a Pt quasispherical $\left(0.055 \mathrm{~cm}^{2}\right.$ area) working electrode placed axially at the top of a cylindrical cell. A large $\mathrm{Pt}$ plate counter-electrode and a saturated calomel reference electrode (sce) were used. Voltammograms show that the $\mathbf{A g}$ electrode behaves as an ideal blocking electrode in $\mathrm{Na}_{2} \mathrm{SO}_{4}+\mathrm{H}_{2} \mathrm{SO}_{4}$ aqueous solutions. These solutions were prepared from a.r. chemicals and Milli*-Q water.

The Ag electrodeposition was made at $-0.200 \mathrm{~V}$ (vs. sce) from $0.01 \mathrm{M} \mathrm{Ag} \mathrm{SO}_{4}+0.01 \mathrm{M} \mathrm{H}_{2} \mathrm{SO}_{4}$ $+0.5 \mathrm{M} \mathrm{Na}_{2} \mathrm{SO}_{4}$ solution[13]. After a preselected Ag electrodeposition charge, $Q$, has been passed, the working electrode was removed from the cell, washed with water, and immersed in the electrochemical cell containing a $x \mathrm{M} \mathrm{H}_{2} \mathrm{SO}_{4}+y \mathrm{M}$ $\mathrm{Na}_{2} \mathrm{SO}_{4}$ solution $\left(10^{-2} \leqslant x \leqslant 10^{-5}, 0.5 \leqslant y \leqslant 5\right.$ $\times\left(0^{-2}\right)$ for the ac impedance measurements. The 
composition range covered by these solutions allowed us to determine the influence of the solution resistance on the impedance response of different $\mathrm{Ag}$ dendritic electrodeposits.

The impedance measurements were performed at $-0.1 \mathrm{~V}$ (vs. sce) using a $0.005 \mathrm{~V}$ signal amplitude in the $1 \mathrm{~Hz} \leqslant f \leqslant 10^{4} \mathrm{~Hz}$ frequency range. These input variables, including the frequency range, provided the maximal reproducible information of the electrochemical system.

The ac impedance measurements were performed with a Solartron FRA 1250 unit coupled to an Electrochemical Interface 1186. A potentiostat EG\&G Model 173 was used for Ag electrodeposition. Runs were made under purified Ar at $25^{\circ} \mathrm{C}$.

\section{RESULTS AND DISCUSSION}

The evolution of $\mathrm{Ag}$ dendritic electrodeposits comprising $Q$ values in the $0<Q<60 \mathrm{mC}$ range is shown in Fig. 1a-c. A sponge-like deposit with a selfsimilar appearance can be seen. The inspection of these deposits at a higher magnification reveals forest-like patterns (Fig. 1d) similar to those already reported for structures fulfilling DLA models[15]. Macroscopically, $\mathrm{Ag}$ deposits can be described as axially symmetric objects, the growing contour having a two symmetry axis, $v$ and $h$ in the vertical and the horizontal directions, respectively (Fig. 2).

The mass $(M)$ vs. volume $(V)$ relationships can be used to characterize volume fractals. In this case, as $M \propto Q$, a $Q$ vs, $V$ plot allows us to characterize the dendritic $\mathrm{Ag}$ three-dimensional deposit. $Q$ was evaluated from $t=0$, the beginning of the $\mathrm{Ag}$ electrodeposition process up to the time $t$ required for the object to reach the volume $V$. Otherwise, $V$ values were calculated by assuming that the growing object can be modeled as a cylinder with height $L_{\mathrm{y}}$ and base diameter $L_{\mathrm{h}}$ (Fig. 2) after correcting for the volume of the $\mathrm{Pt}$ substrate. This volume was attributed to the volume of a sphere of radius $r_{0}$. In this way a
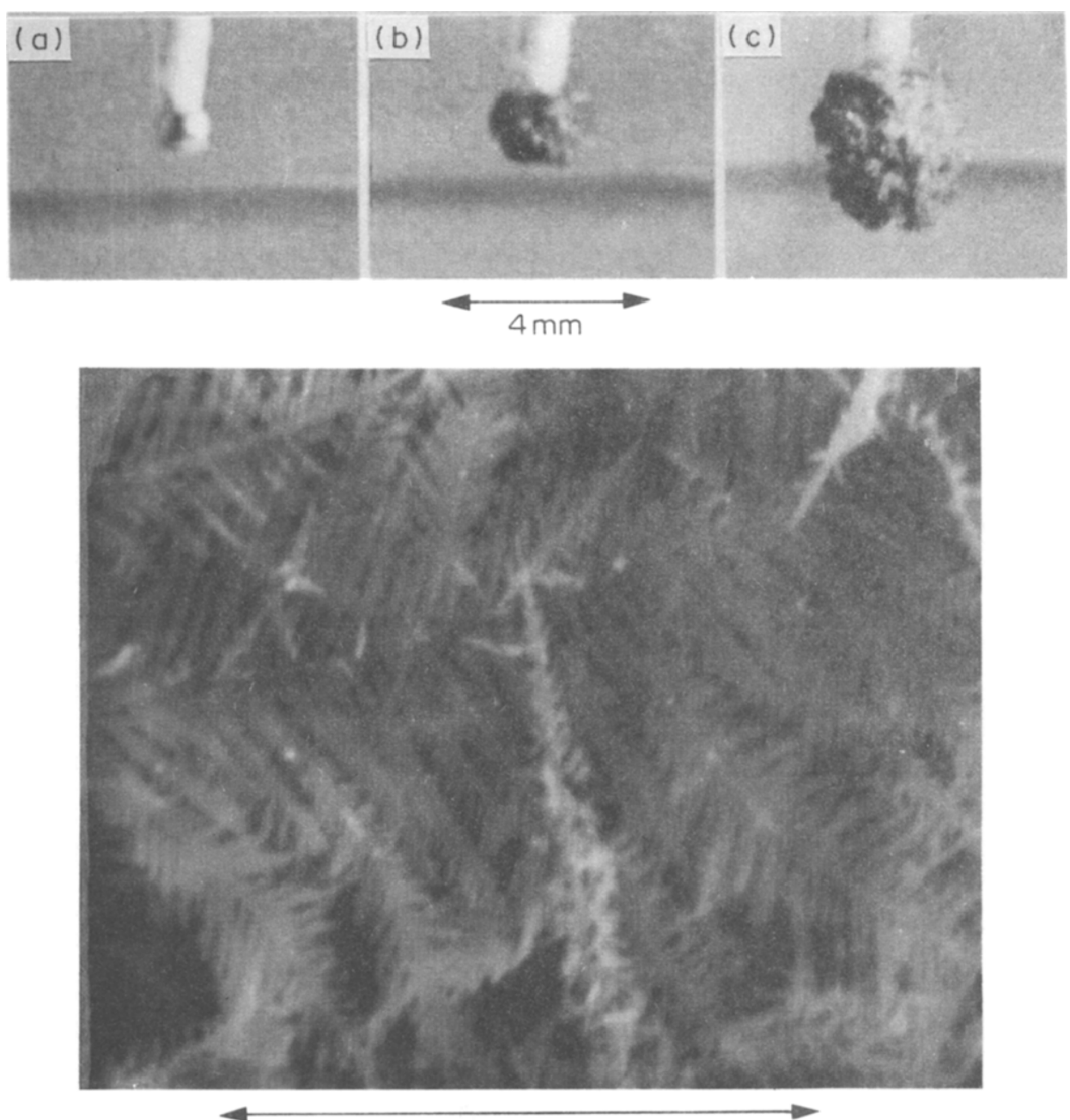

$8 \mu \mathrm{m}$

Fig. 1. (a) Initial single crystal polyfaceted Pt substrate; (b) Three-dimensional Ag dendritic deposit $(Q=10 \mathrm{mC})$; (c) Three-dimensional Ag dendritic deposit $(Q=55 \mathrm{mC})$; (d) SEM micrograph of Ag dendrites. Scale bars are shown in the figure. 


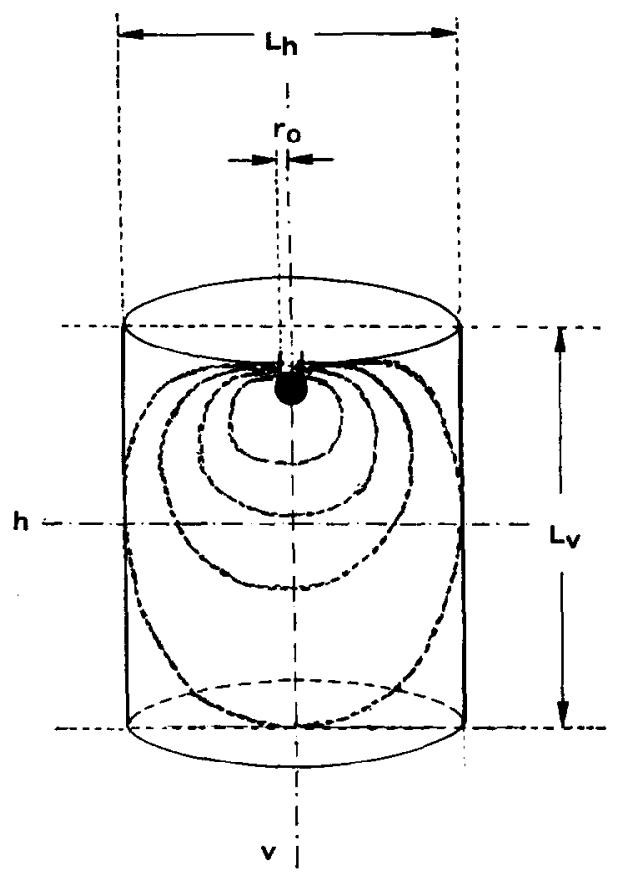

Fig. 2. Geometrical model for scaling the Ag dendritic deposits. Dotted traces represent the contours of the dendritic object at different stages of growth. The quasispherical initial substrate is shown as a black surface.

linear $Q$ vs. $V$ plot was obtained (Fig. 3). From the slope of this straight line $\rho$ value, the apparent density of the $\mathrm{Ag}$ dendritic deposit, results in $\rho=1.66 \times 10^{-3} \mathrm{~g} \mathrm{~cm}^{-3}$, a figure which is slightly higher than the density of the $\mathrm{Ag}^{+}$ions in the solution in which the $\mathbf{A g}$ dendrites were grown. This constant value of $\rho$ indicates that $D_{\mathrm{m}}$, the mass

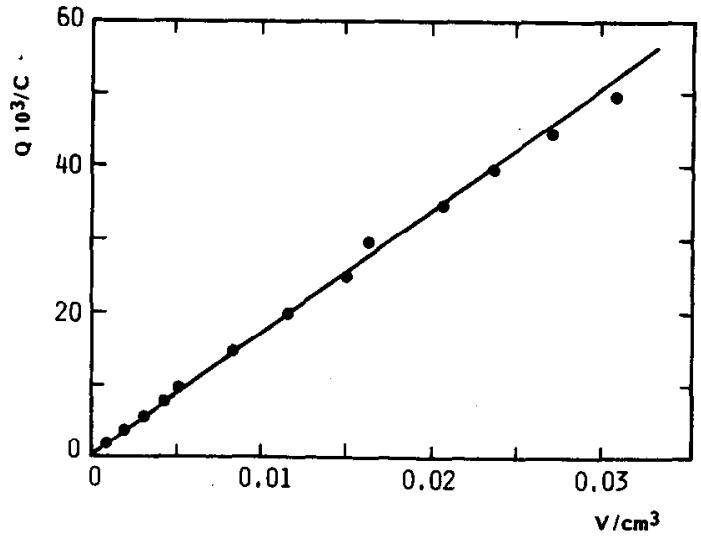

Fig. 3. $Q$ vs. $V$ plot resulting from the three-dimensional Ag dendritic deposit at different stages of growth; $25^{\circ} \mathrm{C}$.

(volume) fractal dimension, is $D_{\mathrm{m}}=3$, ie the growing object behaves as a Euclidean object.

Impedance data of $\mathbf{A g}$ dendritic deposits displayed as $\log Z^{\prime \prime}$ vs. $\log \omega$ plots (Fig. 4) show two broad domains depending on whether $\omega$ is lower or greater than a certain critical frequency $\omega_{c}$. For $\omega<\omega_{c}$ equation (1) is obeyed, in contrast to $\omega>\omega_{c}$. Thus, the $\log Z^{\prime \prime}$ vs. $\log \omega$ plots resulting in $10^{-2} \mathrm{M}$ $\mathrm{Na}_{2} \mathrm{SO}_{4}+2 \times 10^{-4} \mathrm{M} \quad \mathrm{H}_{2} \mathrm{SO}_{4}$ solutions for $Q<8 \mathrm{mC}$ and $\omega<3000 \mathrm{rads}^{-1}$, show a single decreasing straight line portion with an average slope $\langle\alpha\rangle=0.95 \pm 0.03$. In this case, for $\omega>\omega_{c} \cong$ $3000 \mathrm{rads}^{-1}$, a continuous increase in $\log Z^{\prime \prime}$ vs. $\log \omega$ can be observed.

On the other hand, the $\log Z^{\prime \prime}$ vs. $\log \omega$ plots from $\mathrm{Ag}$ deposits in the $8 \mathrm{mC}<Q<60 \mathrm{mC}$ range and $\omega<\omega_{c}$, show two decreasing linear portions defining a crossover frequency at $\omega_{\lambda}$. The $\omega_{\mathrm{c}}$ and $\omega_{\lambda}$ frequency ranges are indicated in Fig. 4. For $\omega<\omega_{\lambda}$,

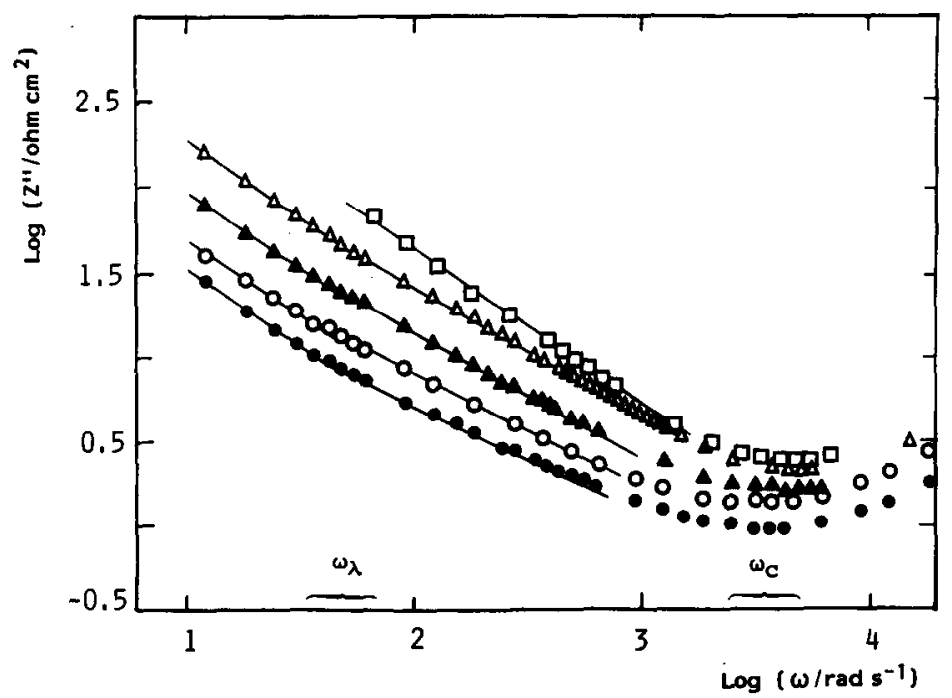

Fig. 4. $\log Z^{\prime \prime}$ vs. $\log \omega$ plot for three-dimensional $\mathrm{Ag}$ dendritic deposits in $10^{-2} \mathrm{M} \mathrm{Na}_{2} \mathrm{SO}_{4}+2$ $\times 10^{-4} \mathrm{M} \mathrm{H}_{2} \mathrm{SO}_{4}$ solutions; (D) $Q=4 \mathrm{mC} ;(\Delta) Q=8 \mathrm{mC} ;(\triangle) Q=16 \mathrm{mC} ;(O) Q=36 \mathrm{mC}$; (O) $Q=60 \mathrm{mC} .25^{\circ} \mathrm{C}$. The $\omega_{\lambda}$ and $\omega_{\mathrm{c}}$ frequency ranges are indicated in the figure. 
the average slope of the linear portion is $\langle\alpha\rangle=0.95 \pm 0.03$; whereas for the $\omega_{\lambda}<\omega<$ $3000 \mathrm{rad} \mathrm{s}^{-1}$ range, $\langle\alpha\rangle=0.67 \pm 0.05$. Furthermore, as $Q$ increases from 8 to $60 \mathrm{mC}$ the trend of the value of $\omega_{\lambda}$ to decrease from 50 to $36 \mathrm{rad} \mathrm{s}^{-1}$ is observed.

Impedance data depend on the electrolyte composition. As the electrolyte concentration is increased from $10^{-2} \mathrm{M} \quad \mathrm{Na}_{2} \mathrm{SO}_{4}+2 \times 10^{-4} \mathrm{M}$ $\mathrm{H}_{2} \mathrm{SO}_{4}$ (Fig. 4) to $0.5 \mathrm{M} \mathrm{Na}_{2} \mathrm{SO}_{4}+10^{-2} \mathrm{M} \mathrm{H}_{2} \mathrm{SO}_{4}$ (Fig. 5), the value of $\omega_{\lambda}$ in the $\log Z^{\prime \prime}$ vs. $\log \omega$ plot increases from $35-65 \mathrm{rad} \mathrm{s}^{-1}$ to $630-1900 \mathrm{rad} \mathrm{s}^{-1}$, but the value $\langle\alpha\rangle=0.67 \pm 0.05$ remains constant. Nevertheless, it should be noted that the frequency range related to the second linear portion in this solution is shorter than at lower solution concentrations. Furthermore, as the electrolyte concentration is increased, the rising portion for $\omega>\omega_{\mathrm{c}}$ is no longer observed (Fig. 5). A similar feature in the $\log Z^{\prime \prime}$ vs. $\log \omega$ plot at high $\omega$ can be observed for a smooth $\mathrm{Pt}$ electrode in $10^{-2} \mathrm{M} \mathrm{Na} \mathrm{Na}_{4}+2$ $\times 10^{-4} \mathrm{M} \mathrm{H}_{2} \mathrm{SO}_{4}$ (Fig. 6). Therefore, this behaviour for $\mathrm{Ag}$ dendrites can hardly be attributed to surface roughness effects.

Note that the ac impedance response of a smooth $\mathrm{Pt}$ electrode in $0.5 \mathrm{M} \mathrm{Na}_{2} \mathrm{SO}_{4}+10^{-2} \mathrm{M} \mathrm{H} \mathrm{H}_{2} \mathrm{SO}_{4}$ shows only one straight line with $\alpha=0.95$. (Fig. 6). Hence, the $\log Z^{\prime \prime}$ vs. $\log \omega$ plot plot for $\omega>\omega_{c}$ appears to be considerably dependent on the electrolyte solution resistance in a way which is still far from being understood. Thence, ac impedance data fulfilling equation (1) will be only considered for the purpose of this work.

The value of $\omega_{\lambda}$ can be used to define a boundary in the $\log Z^{\prime \prime}$ vs. $\log \omega$ plot between the frequency

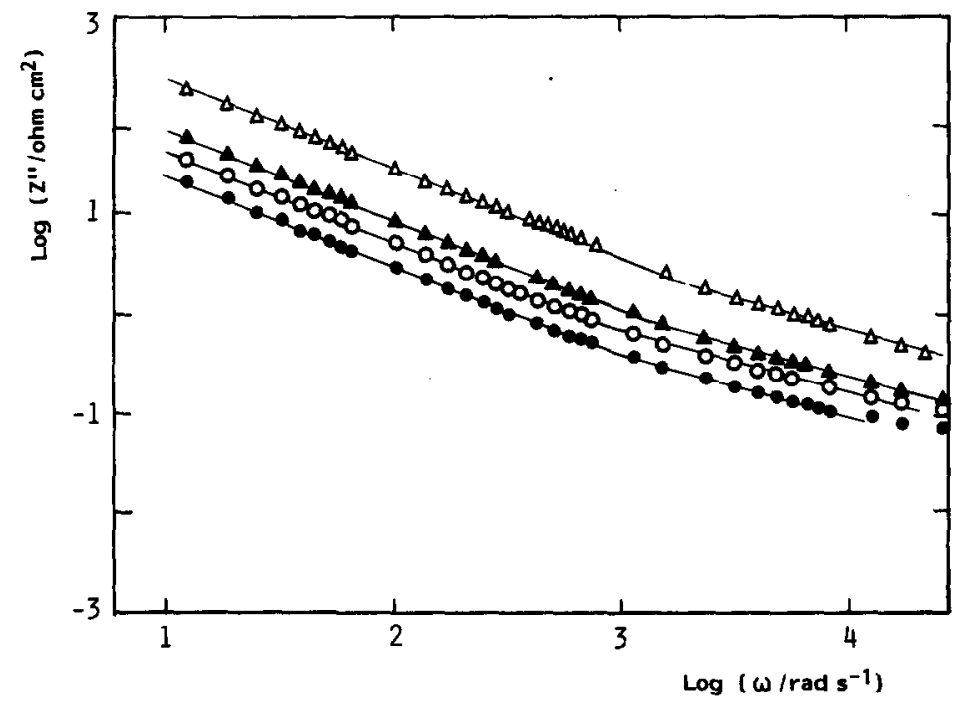

Fig. 5. $\log Z^{\prime \prime}$ vs. $\log \omega$ plot for three-dimensional $\mathrm{Ag}$ dendritic deposit in $0.5 \mathrm{M} \mathrm{Na}_{2} \mathrm{SO}_{4}+2 \times 10^{-4} \mathrm{M}$ $\mathrm{H}_{2} \mathrm{SO}_{4} ;(\triangle) Q=16 \mathrm{mC} ;(\Delta) Q=20 \mathrm{mC} ;(\mathrm{O}) Q=36 \mathrm{mC} ;(O) Q=60 \mathrm{mC} .25^{\circ} \mathrm{C}$..

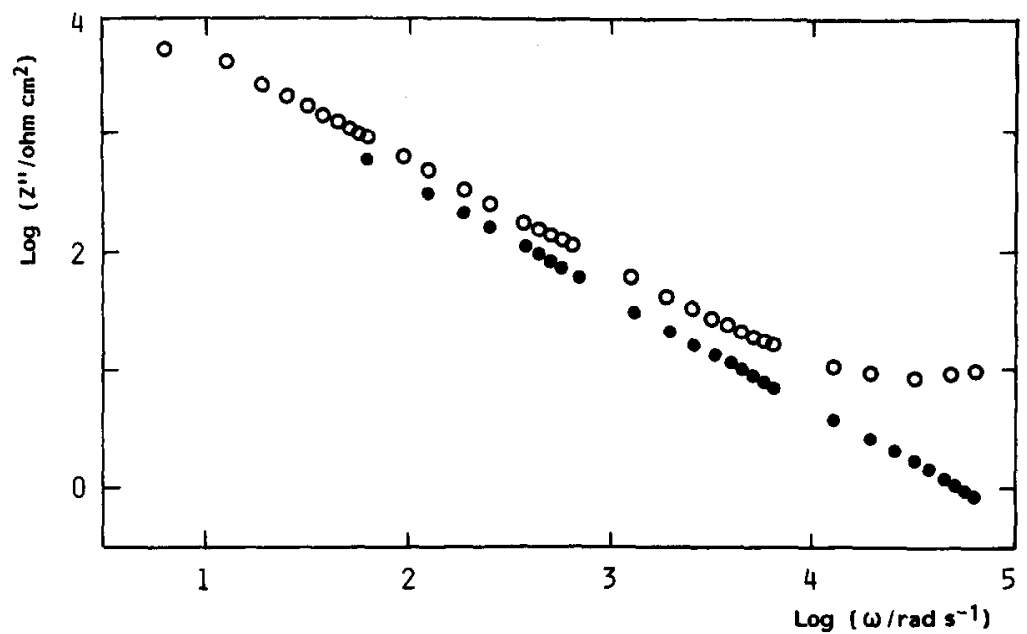

Fig. 6. $\log Z^{n}$ vs. $\log \omega$ plot for a smooth polyfaceted $\mathrm{Pt}$ single crystal in (O) $10^{-2} \mathrm{M} \mathrm{Na} \mathrm{SO}_{4}+2$ $\times 10^{-4} \mathrm{M} \mathrm{H}_{2} \mathrm{SO}_{4}$ at $25^{\circ} \mathrm{C}$ and (O) $0.5 \mathrm{M} \mathrm{Na}_{2} \mathrm{SO}_{4}+10^{-2} \mathrm{M} \mathrm{H}_{2} \mathrm{SO}_{4}$. 
domains involving $\langle\alpha\rangle \cong 0.95 \pm 0.03$ and $\langle\alpha\rangle \cong 0.67 \pm 0.05$. A relationship between $\omega_{\lambda}$ and $\lambda_{c}$, the critical penetration length of the electric signal, has been found for $\mathrm{Ag}$ dendritic deposits [4, 16].

$$
\lambda_{\mathrm{c}}=1 / \rho \omega C_{\mathrm{d}}
$$

where $\rho$ is the resistivity of the electrolyte solution and $C_{\mathrm{d}}$ is the specific capacitance of the electrochemical interface.

Attempts made to estimate $\lambda_{c}$ from equation (3) for $\omega=\omega_{2}$, using $\omega_{2}$ values derived from Figs 4 and $5, C_{\mathrm{d}}=5 \times 10^{-5} \mathrm{~F} \mathrm{~cm}^{-2}, \rho \cong 500 \Omega \mathrm{cm}$ for $10^{-2} \mathrm{M}$ $\mathrm{Na}_{2} \mathrm{SO}_{4}$, and $\rho \cong 10 \Omega \mathrm{cm}$ for $0.5 \mathrm{M} \mathrm{Na}_{2} \mathrm{SO}_{4}$, result in values of $\lambda_{c}$ nearly one decade greater than the size of three-dimensional $\mathrm{Ag}$ dendritic deposits. Equation (3), which has been successfully applied to volume fractals, appear to be insufficient to render a quantitative interpretation of these data.

The CPA behaviour resulting from the ac impedance data of three-dimensional $\mathrm{Ag}$ dendrites can be explained in terms of a uniform mass (volume) object presenting fractal surface characteristics[13, 14]. Seemingly, the very little dependence of $\omega_{2}$ on $Q$ is consistent with a surface fractal object.

Presently, there is no agreement about the validity of parametric relationships between $\alpha$ and $D$, for rough metal surfaces[11]. However, it has been recently proved that equation (2) holds for a DLAlike electrode[12]. If one assumes that equation (2) is valid for three-dimensional Ag dendritic deposits, then it results in $D_{\mathrm{s}}=2.45 \pm 0.1$. This figure coincides with the value of $D_{3}$ for the surface of threedimensional Ag dendritic deposits previously determined[13]. It should be noted that equation (2) has fulfilled hest the correlation between $\alpha$ and $D_{\mathrm{s}}$ for two-dimensional $\mathrm{Ag}$ dendritic deposits grown in a pseudo-two-dimensional electrochemical cell[17].

Acknowledgements-This work was developed within the frame of the Programa de Cooperación Cientifica con lberoamérica (1991), Ministerios de Educación y Ciencias y de Asuntos Exteriores, Spain.

\section{REFERENCES}

1. R. de Levie, Electrochim. Acta 10, 113 (1965).

2. W. Scheider, J. phys. Chem. 79, 127 (1975).

3. L. Nyikos and T. Pajkossy, Electrochim. Acta 30, 1533 (1985).

4. B. Sapoval, J. Chazalviel and J. Peyrière, Phys. Rev. A 38, 5867 (1988).

5. M. Keddam and H. Takenouti, C. R. Acad. Sci. 302, 281 (1986).

6. R. de Levie, J. electroanal. Chem. 281, 1 (1990).

7. S. H. Liu, Phys. Rev. Lett. 55, 529 (1985).

8. A. Le Mehauté and G. Crepy, Solid State Ionics 9/10, 17 (1983).

9. T. C. Halsey, Phys. Rev. A 36, 5877 (1987).

10. R. Ball and M. Blunt, J. Phys. A 21, 197 (1988).

11. T. Pajkossy, J. electroanal. Chem. 300, 1 (1991).

12. P. Meakin and B. Sapoval, Phys. Rev. A 43, 2993 (1991).

13. A. Hernảndez-Creus, P. Carro, S. González, R. C. Salvarezza and A. J. Arvia, J. electrochem. Soc. 139, 1064 (1992).

14. R. M. Brady and R. C. Ball, Nature 309, 225 (1984).

15. M. Matsushita, in The Fractal Approach to Heterogeneous Chemistry (Edited by D. Avnir) p. 161. Wiley, New York (1989).

16. E. Chassaing, B. Sapoval, G. Daccord and R. Lenormand, J. electroanal. Chem. 279, 67 (1990).

17. G. Kahanda and M. Tomkiewicz, J. electrochem. Soc. 137, 3423 (1990). 